\title{
Percentage body fat and metabolic health in Irish adolescents - preliminary analysis from the National Teens' Food Survey II
}

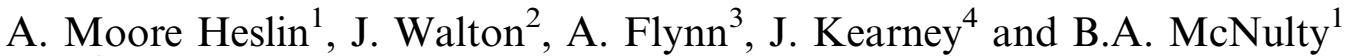 \\ ${ }^{1}$ UCD Institute of Food and Health, University College Dublin, Dublin, Ireland, \\ ${ }^{2}$ Department of Biological Sciences, Munster Technological University, Cork, Ireland, \\ ${ }^{3}$ School of Food and Nutritional Sciences, University College Cork, Cork, Ireland and \\ ${ }^{4}$ School of Biological \& Health Sciences, Technological University Dublin, Dublin, Ireland
}

Obesity is a major risk factor for the onset of cardiovascular disease and metabolic dysfunction. The growing prevalence of adolescent obesity raises concerns regarding metabolic health amongst teenagers, with existing research indicating an increasing presentation of cardiometabolic risk factors such as dyslipidemia, hyperglycemia, and inflammation within adolescents with obesity ${ }^{(1,2)}$. The aim of this study was to investigate associations between percentage body fat and metabolic health parameters, namely blood lipids and inflammatory biomarkers, in Irish adolescents.

Analyses are based on a subsample of data collected from the National Teens' Food Survey II (2019-2020) (www.iuna.net) $(\mathrm{n}=246,50 \%$ male). Percentage body fat $(\% \mathrm{BF})$ was measured via bioelectrical impedance using a Tanita BC-420MA body composition analyzer. Participants were stratified according to age and gender specific \%BF z-score tertiles. Serum lipids and high sensitivity $\mathrm{C}$ reactive protein (hsCRP) were measured via bioanalyzer. Visceral adiposity was estimated using the visceral adiposity index ${ }^{(3)}$. Pubertal development stage was estimated using urinary creatinine excretion ${ }^{(4,5)}$. Covariate adjusted general linear model and linear regression were used assess relationships between $\% \mathrm{BF}$ and metabolic health parameters, with age, gender, fasting state, medication use, physical activity and pubertal development stage included as covariates in all analysis.

Overall, $11 \%$ of the subsample were classed as having obesity and $9 \%$ as having overfat based on age and gender-specific $\% \mathrm{BF}$ cut-offs ${ }^{(6)}$. Assessment of blood lipids across $\% \mathrm{BF}$ z-score tertiles revealed significantly increased mean serum triglycerides amongst the highest $\% \mathrm{BF}$ tertile $(0.79 \mathrm{mmol} / \mathrm{L} \pm 0.45)$ compared to the lowest $\% \mathrm{BF}$ tertile $(0.59 \mathrm{mmol} / \mathrm{L} \pm 0.22)(\mathrm{p}<0.001, \eta \mathrm{p} 2=0.066)$. Increased body fat was significantly associated with increases in serum calculated LDL cholesterol $(\beta=0.350,95 \%$ CI $(0.065-0.140) p<0.001)$ with $21 \%$ of participants within the highest $\% \mathrm{BF}$ z-score tertile displaying elevated LDL as determined by NCEP cut offs ${ }^{(7)}$ compared to $5 \%$ of those within the lowest $\% \mathrm{BF}$ z-score tertile $(\mathrm{p}=0.019)$. $\% \mathrm{BF}$ z-score was inversely associated with HDL cholesterol $(\beta=-0.242,95 \%$ CI $(-0.020--0.006), p<0.001)$, with an associated increase in blood lipid ratios observed amongst the highest $\% \mathrm{BF}$ tertile. A significant stepwise increase in visceral adiposity index score was observed amongst increasing \% $\mathrm{BF} \mathrm{z}$ score tertiles $(\mathrm{p}<0.001, \eta \mathrm{p} 2=0.108)$. A higher $\% \mathrm{BF}$ z-score was significantly associated with elevated inflammation as indicated by serum hsCRP $(\beta=0.277,95 \%$ CI $(0.046-0.129) \mathrm{p}<0.001)$.

Adolescents with increased body fat display a less favorable blood lipid profile and indication of elevated inflammation, with elevations in serum triglycerides and hsCRP, an increased prevalence of clinically elevated LDL cholesterol and a tendency towards decreased HDL cholesterol. The results of this analysis indicate a possible trend towards a more unfavorable metabolic profile amongst adolescents with increased body fat. Future research analyzing additional metabolic parameters and examining the dietary and environmental factors relating to adolescent obesity and metabolic health are warranted.

\section{Acknowledgements}

Many thanks to the participants, fieldworkers and staff of NTFSII. This research was funded by the Irish Department of Agriculture, Food and the Marine under the project 'National Teens' Food Consumption Survey II'.

\section{References}

1. Dai S, et al. (2009) Am J Prev Med

2. Ruiz LD, et al. (2020) Nutrients

3. Amato MC \& Giordano C. (2014) Int J Endocrinol

4. Zheng $\mathrm{H}$, et al. (2014) Biomed Res Int

5. Singh GKS, et al. (2015) Ann Clin Biochem

6. McCarthy HD, et al. (2006) Int J Obes

7. Lauer RM, et al. (1992) Pediatrics 